\title{
Usual Interstitial Pneumonia Pattern Interstitial Lung Disease Developed in a Patient with IgG4-related Chronic Sclerosing Sialadenitis: A Case Report
}

\author{
Hiroshi Yamamoto ${ }^{1}$, Masamichi Komatsu ${ }^{1}$, Kei Sonehara ${ }^{1}$, Yuichi Ikuyama ${ }^{1}$, \\ Kazuhisa Urushihata ${ }^{1}$, Kazunari Tateishi ${ }^{1}$, Yoshiaki Kitaguchi ${ }^{1}$, Atsuhito Ushuiki ${ }^{1}$, \\ Shiho Asaka ${ }^{2}$, Takeshi Uehara ${ }^{2}$, Satoshi Kawakami ${ }^{3}$, Kentaro Mori ${ }^{4}$, Kazutoshi Hamanaka ${ }^{5}$, \\ Kenichi Nishie $^{6}$, Akira Hebisawa ${ }^{7}$ and Masayuki Hanaoka ${ }^{1}$
}

\begin{abstract}
:
A 69-year-old man was diagnosed with immunoglobulin (Ig) G4-related disease (IgG4-RD) at 62 years old. At that time, he had high serum IgG4 levels and bilateral submandibular gland swelling on CT; thus, a gland biopsy was performed. Because a reticular shadow was found on chest CT, a lung surgical biopsy was also performed. The specimens revealed usual interstitial pneumonia (UIP) pattern interstitial pneumonia with some IgG4-positive cells. The patient was subsequently followed up without treatment. His forced vital capacity and radiological findings progressively deteriorated, consistent with UIP pattern interstitial lung disease but different from a lung lesion of IgG4-RD.
\end{abstract}

Key words: immunoglobulin (Ig) G4, immunoglobulin (Ig) G4-related disease (IgG4-RD), progressive fibrosing, interstitial pneumonia, usual interstitial pneumonia (UIP)

(Intern Med Advance Publication)

(DOI: 10.2169/internalmedicine.8937-21)

\section{Introduction}

Immunoglobulin (Ig) G4-related disease (IgG4-RD) is a systemic disease characterized by tumefactive lesions with abundant IgG4-positive plasma cells and high serum IgG4 concentrations (1-4). In the respiratory lesions of IgG4-RD, four major categories (solid nodular type, round-shaped ground-glass opacity (GGO) type, alveolar interstitial type with honeycombing, and bronchovascular type) have been reported (5). We previously reported 18 patients with IgG4related respiratory disease (IgG4-RRD), which is characterized by respiratory involvement of IgG4-RD, with extrathoracic manifestations of IgG4-RD (6). All 18 patients had a benign prognosis. Interestingly, none of the 18 patients showed alveolar interstitial type with honeycombing. Thus, we wondered if a lung manifestation of IgG4-RD, such as alveolar interstitial type with honeycombing, actually existed.

We herein report a patient already diagnosed with IgG4$\mathrm{RD}$ of the submandibular glands who showed a reticular shadow on chest CT and who underwent a lung surgical biopsy.

\section{Case Report}

A 62-year-old man with few respiratory symptoms was referred by the otolaryngology department for the examina-

${ }^{1}$ First Department of Internal Medicine, Shinshu University School of Medicine, Japan, ${ }^{2}$ Department of laboratory medicine, Shinshu University School of Medicine, Japan, ${ }^{3}$ Department of Radiology, Shinshu University School of Medicine, Japan, ${ }^{4}$ Department of Otorhinolaryngology, Shinshu University School of Medicine, Japan, ${ }^{5}$ Department of Surgery, Division of General Thoracic Surgery, Shinshu University School of Medicine, Japan, ${ }^{6}$ Department of Respiratory Medicine, Iida Municipal Hospital, Japan and ${ }^{7}$ Division of Clinical Pathology, Asahi General Hospital, Japan

Received: October 30, 2021; Accepted: December 20, 2021; Advance Publication by J-STAGE: February 8, 2022

Correspondence to Dr. Masamichi Komatsu, mskomatsu@shinshu-u.ac.jp 

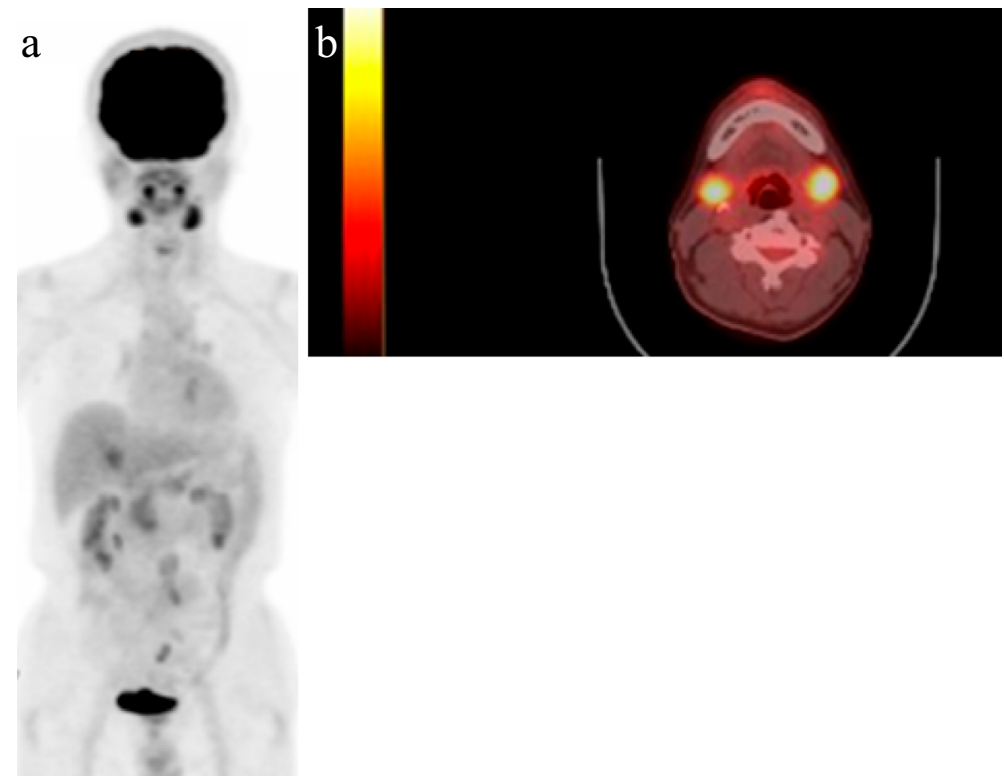

Figure 1. Fluorine-18 fluorodeoxyglucose-positron emission tomography (FDG-PET) showed bilateral swollen submandibular glands and the abnormal accumulation of FDG in the glands [(a) whole-body view; (b) lachrymal glands].
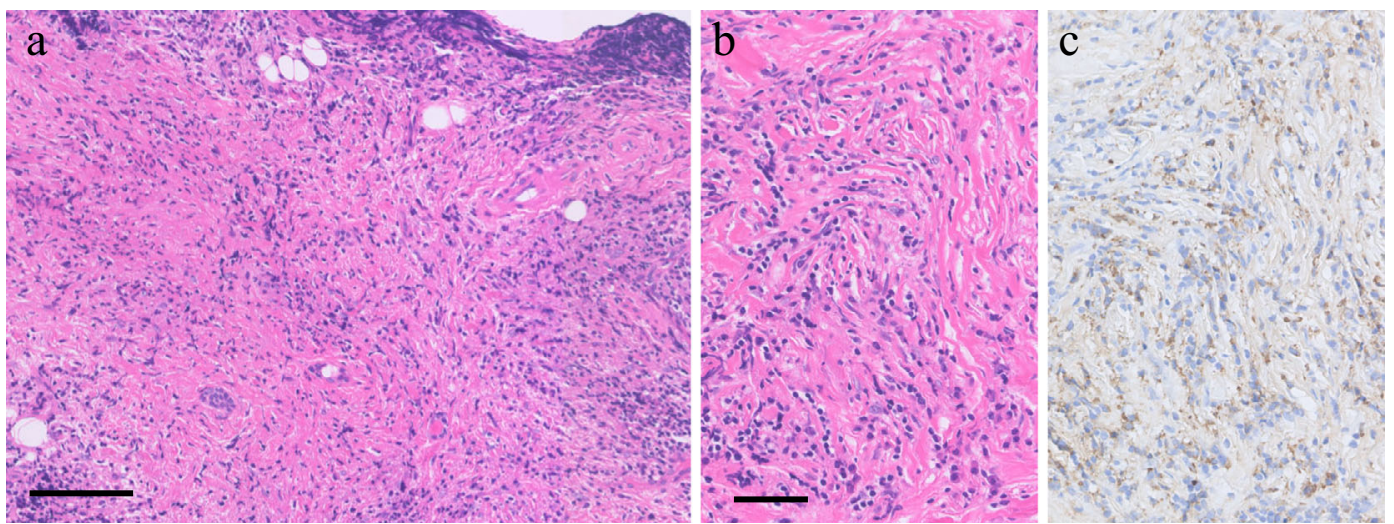

Figure 2. The left mandibular gland biopsy specimen showed chronic sialadenitis with a storiform pattern of fibrosis [Hematoxylin and Eosin (H\&E) staining, scale bar=100 $\mu \mathrm{m}$ ] (a). An immunohistochemical examination of the specimen revealed increased infiltration of IgG4-positive plasma cells [>50/high-power field (HPF)] [H\&E staining (b) and IgG4 immunostaining (c), scale bar=50 $\mu \mathrm{m}]$.

tion of a lesion and visited our respiratory department (Shinshu University Hospital, Matsumoto, Japan). He had no family history of pancreatic disease, collagen disease, or autoimmune disease. He had no history of smoking, occupational inhalation, or respiratory diseases. Two months before coming to our division, he was diagnosed with IgG4-RD of the submandibular glands based on the comprehensive diagnostic criteria for IgG4-RD (1). The patient had a high serum level of $\operatorname{IgG} 4(>135 \mathrm{mg} / \mathrm{dL})$ and swelling of bilateral submandibular glands. The fluorine-18 fluorodeoxyglucosepositron emission tomography (FDG-PET)/CT revealed swelling of the bilateral submandibular glands and an abnormal FDG accumulation in the bilateral submandibular glands (Fig. 1).

A left mandibular gland biopsy was performed, and the specimen showed chronic sialadenitis with a storiform pattern of fibrosis (Fig. 2a). An immunohistochemical examination of the specimen revealed increased infiltration of IgG4positive plasma cells (IgG4-positive cells >10/high-power field $[\mathrm{HPF}]$ and IgG4-positive/IgG-positive cell ratio $>40 \%$ ) (Fig. 2b, c). Furthermore, reticular shadows of bilateral lungs were noted in a radiologist's diagnostic report of the FDG-PET/CT findings.

At the first visit to our division, the patient was $145.2 \mathrm{~cm}$ tall and weighed $40.5 \mathrm{~kg}$. His peripheral oxygen saturation $\left(\mathrm{SpO}_{2}\right)$ was $98 \%$, and fine crackles were heard in his bilateral lower chest.

Laboratory examinations showed the following values, seen in a table below (normal range): peripheral white blood cell count, 8,080/ $\mu \mathrm{L}$; hemoglobin $(\mathrm{Hb}), 12.9 \mathrm{~g} / \mathrm{dL}$; platelet 
Table. Laboratory Data and Pulmonary Function Tests on Admission.

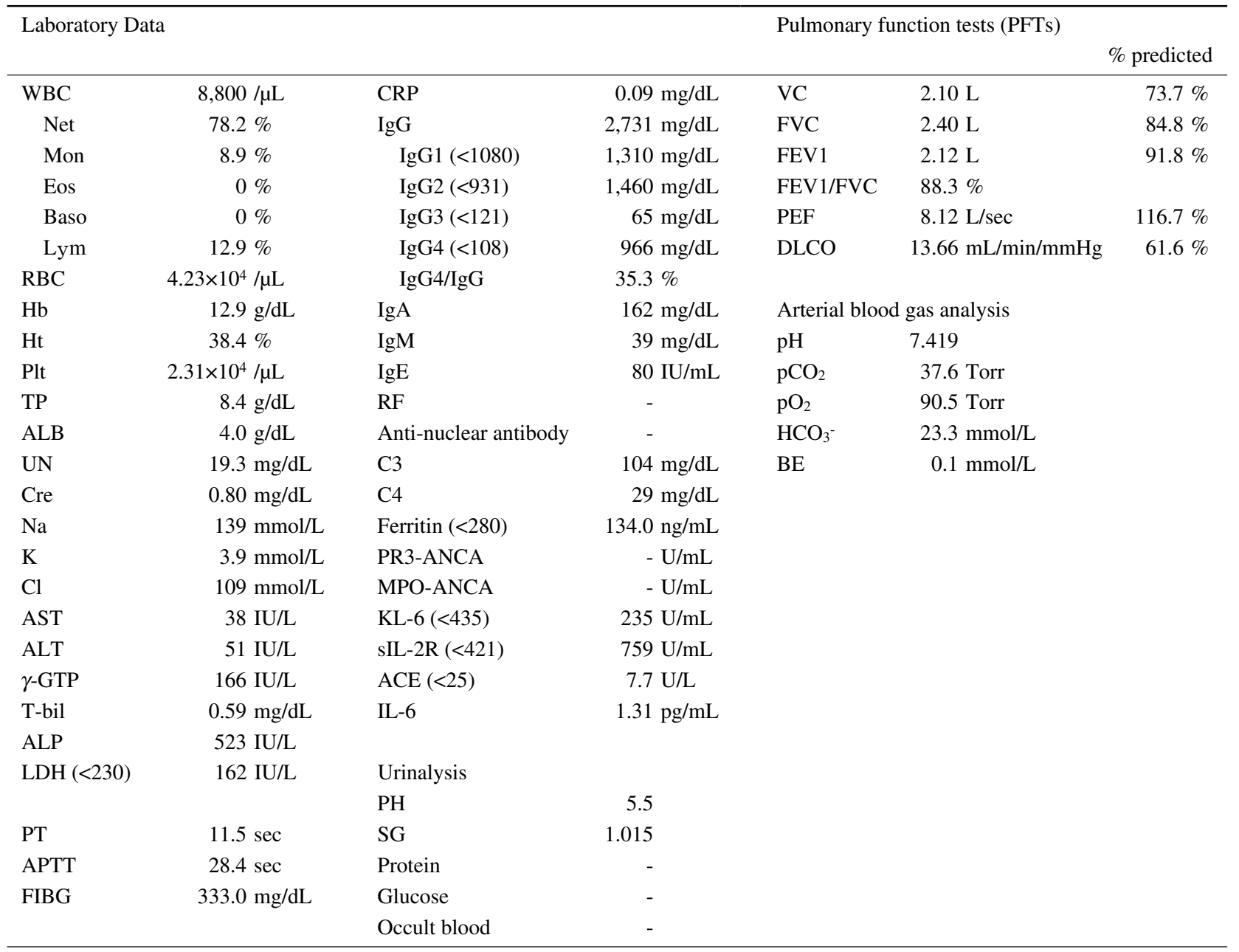

sIL-2R: soluble interleukin-2 receptor, VC: vital capacity, ACE: angiotensin-converting enzyme, FVC: forced vital capacity, KL-6: Krebs von den Lungen-6, FEV1: forced expiratory volume in one second, PEF: peak expiratory flow, DLCO: diffusing capacity of the lung for carbon monoxide

(PLT), $23.1 \times 10^{4} / \mu \mathrm{L}$; total protein $(\mathrm{TP}), 8.4 \mathrm{~g} / \mathrm{dL}$; albumin, $4.0 \mathrm{~g} / \mathrm{dL}$; lactate dehydrogenase (LD), $162 \mathrm{IU} / \mathrm{L}(<220)$; Creactive protein, $0.09 \mathrm{mg} / \mathrm{dL}$; Krebs von den Lungen-6 (KL6), $235 \mathrm{U} / \mathrm{mL}(<435) ; \mathrm{IgG}, 2,731 \mathrm{mg} / \mathrm{dL}(<1,700)$; and $\mathrm{IgG}$ 4, $966 \mathrm{mg} / \mathrm{dL}(<108)$. The serum autoantibodies were all negative. Pulmonary function tests (PFTs) revealed the following values, seen in a table below: a vital capacity (VC) of $2.10 \mathrm{~L}$ ( $73.7 \%$ predicted), a forced vital capacity (FVC) of $2.40 \mathrm{~L}$ ( $84.8 \%$ predicted), and a diffusing capacity of the lung for carbon monoxide ( $\mathrm{DL}_{\mathrm{co}}$ ) of $13.66 \mathrm{~mL} / \mathrm{min} / \mathrm{mmHg}$ (61.6\% predicted).

Chest X-ray showed a reticular shadow in the bilateral lower lung fields (Fig. 3a). Chest CT revealed bilateral ground-glass and reticular opacities predominantly in the lower and peripheral lung zones (Fig. 3b-d). We obtained surgical lung biopsy specimens of the left lung (segment [S] 4, S6, S8), which revealed chronic fibrosing interstitial pneumonia with a usual interstitial pneumonia (UIP) pattern (Fig. 4a) (7, 8). An immunohistochemical examination showed some IgG4-positive plasma cell infiltration ( $>10 /$ $\mathrm{HPF}$ and IgG4-positive cells/IgG-positive cells >40\%) surrounding the lymphoid follicle near the fibroblastic foci; however, neither obliterative vasculitis nor storiform fibrosis was observed, which are characteristic findings of IgG4-RD (Fig. 3b, c) (1-3). According to the proposed diagnostic criteria for IgG4-RRD (10), this case corresponded to "definite" IgG4-RRD. However, as the pathological findings of the patient showed UIP pattern interstitial pneumonia, we were unable to determine whether or not this lung lesion could be considered lung involvement of IgG4-RD or another interstitial disease that developed independently of $\mathrm{IgG}$ 4-RD. The patient had few respiratory symptoms and had no desire for treatment; thus, we decided to follow the patient according to idiopathic pulmonary fibrosis (IPF) at that time.

The patient was then carefully followed without treatment at another hospital. He became aware of mild shortness of breath, only during exertion. His PFT results showed changes over time; his FVC values at 65, 67, and 69 years of age were $2.23 \mathrm{~L}$ (75.9\% predicted), $2.09 \mathrm{~L}$ (77.2\% predicted), and $1.83 \mathrm{~L}(69.0 \%$ predicted), respectively.

At 69 years old, chest X-ray and chest CT were performed. Chest X-ray showed the progression of the reticular shadow in the bilateral lower lung fields with reduced capacity (Fig. 5a). Chest CT revealed deteriorated bilateral ground-glass and reticular opacities, traction bronchiectasis, 

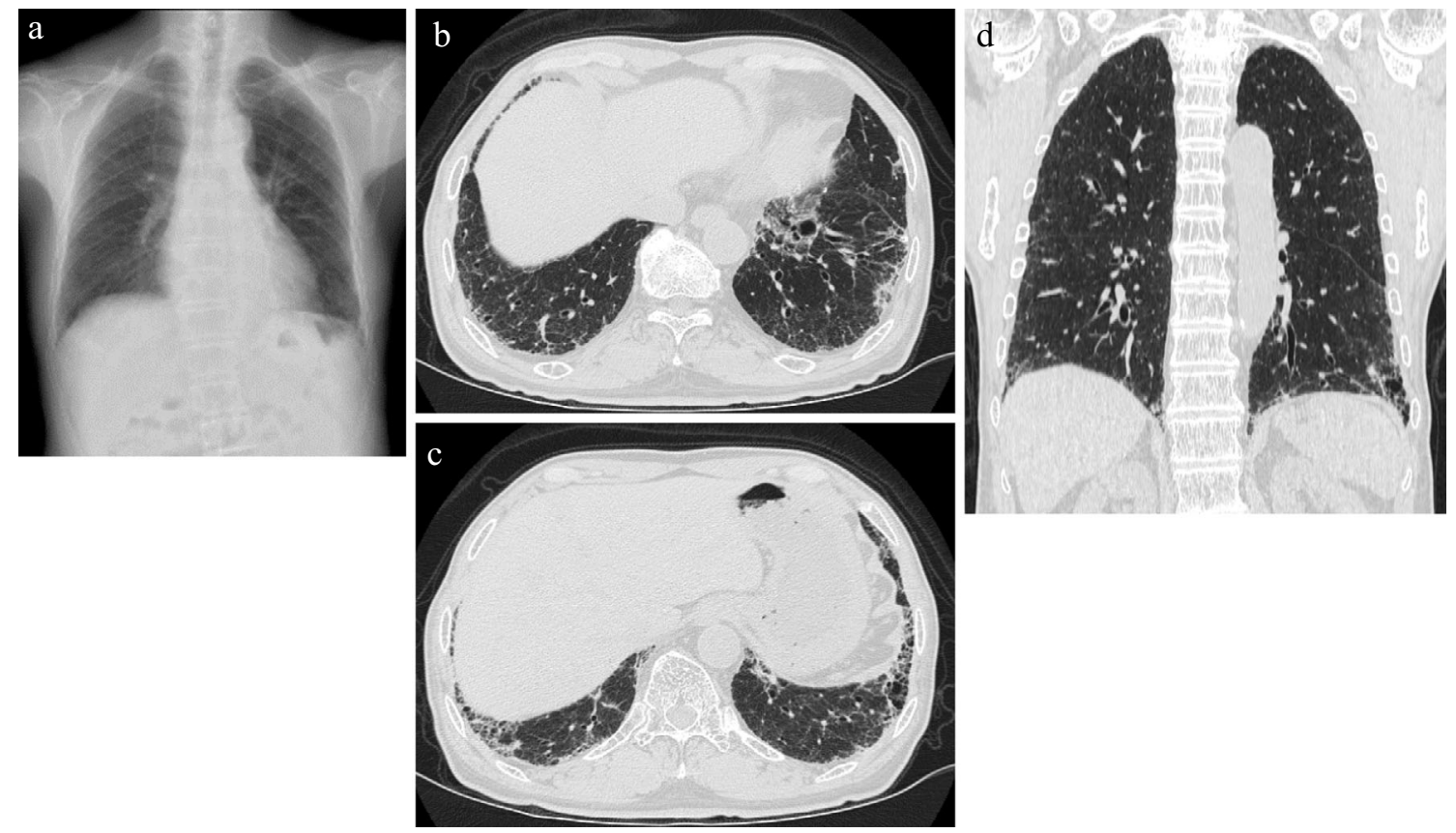

Figure 3. Chest X-ray at 62 years old showed reticular shadow in the bilateral lower lung fields (a). Chest CT at 62 years old revealed bilateral ground-glass and reticular opacities, predominantly in the lower and peripheral lung zones (b-d).
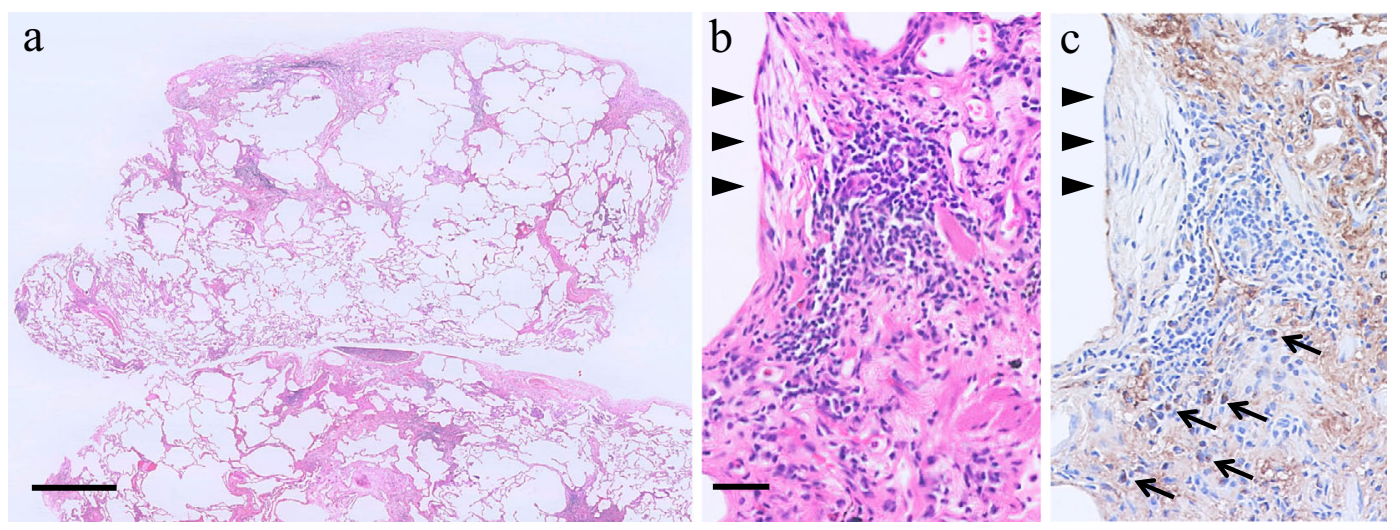

Figure 4. The surgical lung biopsy specimen showed chronic interstitial pneumonia with a usual interstitial pneumonia pattern [Hematoxylin and Eosin (H\&E) staining, scale bar=1 mm] (a). An immunohistochemical examination of the specimen revealed a certain amount of IgG4-positive cell infiltration [>10/high-power field (HPF)] (arrows) surrounding the lymphoid follicle near the fibroblastic foci (arrowheads) [H\&E staining (b) and IgG4 immunostaining (c), scale bar=50 $\mu \mathrm{m}]$.

and honeycombing, predominantly in the lower and peripheral lung zones (Fig. 5b-d). Due to the apparent deterioration of the radiological findings and FVC, the lung lesions were considered consistent with the clinical course of UIP pattern interstitial lung disease (ILD), such as IPF, and showed a progressive fibrosing phenotype (9).

\section{Discussion}

We reported an elderly man with IgG4-RD who was pathologically diagnosed with UIP pattern interstitial pneumonia with some IgG4-positive cell infiltration at 62 years old. After following the patient for seven years without medica- tion, the patient showed a significant decrease in FVC along with a decline in his chest CT findings.

The patient was diagnosed with submandibular grands involvement of IgG4-RD based on the comprehensive diagnostic criteria for IgG4-RD (1). Because the patient had elevated serum IgG4 levels, an extrathoracic lesion of IgG4$\mathrm{RD}$, bilateral infiltrative shadow on chest CT, and some IgG 4-positive plasma cell infiltration ( $>10 / \mathrm{HPF}$ and IgG4positive cells/IgG-positive cells $>40 \%$ ) in the lung biopsy specimen, his lung lesion met the diagnostic criteria for IgG 4-RRD (10). IgG4-RRD develops through the lymphatic routes with abundant IgG4-positive cells in the lungs and responds well to corticosteroid therapy with a benign progno- 


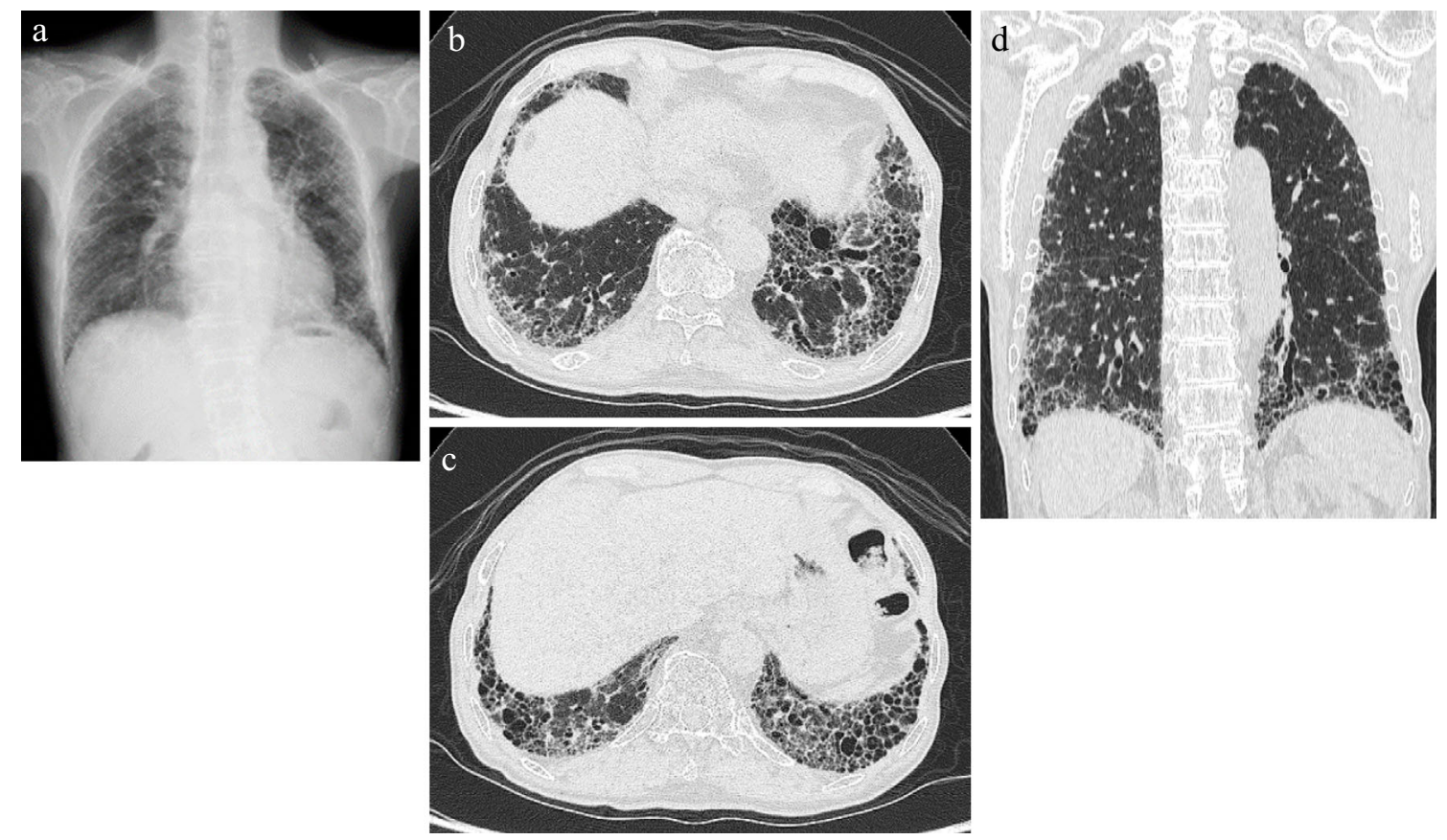

Figure 5. Chest X-ray at 67 years old showed progression of the reticular shadow in the bilateral lower lung fields with reduced capacity (a). Chest CT at 67 years old revealed bilateral deteriorated ground-glass and reticular opacities, traction bronchiectasis, and honeycombing, predominantly in the lower and peripheral lung zones (b-d).

sis $(6,11,12)$. Because the UIP pattern interstitial pneumonia of the patient did not show obliterative vasculitis and storiform fibrosis, which are characteristic findings of IgG4$\mathrm{RD}$, the pathological findings of the lung lesion were considered inconsistent with those of IgG4-RRD developing through the lymphatic routes (6). Furthermore, the lung lesion of the patient deteriorated slowly but progressively, and honeycombing became apparent. This clinical course and the progression of the CT findings of this lung lesion were considered consistent with UIP pattern ILD (e.g. IPF) and differed from IgG4-RRD (6).

Recently, we reported 16 patients with IgG4-positive interstitial pneumonia (IP) who showed abundant IgG4positive cells in the lungs and elevated serum IgG4 levels without extrathoracic lesions of IgG4-RD (13). Histopathologically, marked numbers of IgG4-positive cells in the fibrous parenchyma were observed in 14 patients. However, none of these 16 patients had either obliterative vasculitis or storiform fibrosis. The characteristic chest CT findings of IgG4-positive IP, which differed somewhat from those of IgG4-RRD, as we previously reported (6), were groundglass opacities $(n=16)$, reticular opacities $(n=11)$, traction bronchiectasis $(n=10)$, thickening of the interlobular septal wall $(n=9)$, and hilar mediastinal lymphadenopathy $(n=6)$. We detected a correlation between the distribution of ground-glass opacities on chest CT and the lymphoplasmacytic inflammation in the lung tissue. Furthermore, a correlation existed between the patterns of traction bronchiectasis and reticular opacities on chest CT and histopathological fibrosis. All 15 patients with ground-glass opacities on chest CT who received steroid treatment showed improvement. In contrast, the reticular opacities deteriorated after the initiation of treatment in 6 of the 15 patients. Despite various treatments, three patients died (two patients due to chronic respiratory failure and one due to an acute exacerbation). We speculate that the improvement in ground-glass opacities on chest CT reflected the elimination of IgG4-positive cell infiltration from the perilymphatic stromal area in the lung. Residual fibrosis may have been the essence of IP leading to progressive fibrosis, even though the ground-glass opacities improved with corticosteroid treatment. We conclude that IgG4-positive IP should be treated as a different category from conventional IgG4-RRD because of the significant differences in disease behavior and responses to corticosteroid therapy.

The 2019 American College of Rheumatology (ACR)/ European League Against Rheumatism (EULAR) classification criteria for IgG4-RD were formulated from rheumatismrelated academic societies (14). In the ACR/EULAR classification criteria, entry criteria, exclusion criteria, and inclusion criteria were clarified. In particular, poor responsiveness to corticosteroid therapy is emphasized in the exclusion criteria. Most patients with IgG4-positive IP are likely excluded from IgG4-RD by the ACR/EULAR classification criteria due to poor responsiveness to corticosteroid therapy. Furthermore, the 2020 revised comprehensive diagnostic (RCD) criteria for IgG4-RD also state that the diagnosis should be reconsidered in cases of poor steroid responsiveness (15). Most patients with IgG4-positive IP are likely also excluded from IgG4-RD according to the 2020 RCD criteria for IgG 4-RD.

The four types of lung lesions of IgG4-RD that were first 
reported (solid nodular, round-shaped GGO, alveolar interstitial with honeycombing, and bronchovascular type) were based on mainly radiological and pathological findings (5). By contrast, the clinical course, such as responsiveness to corticosteroid therapy, has been less thoroughly explored. Based on recent criteria for $\operatorname{IgG} 4-\operatorname{RD}(14,15)$, we believe that it is essential to reassess the lung lesions of IgG4-RD, including their clinical course (e.g. treatment responsiveness). We therefore suggest that alveolar interstitial-type lung lesions with honeycombing, many of which fall under the definition of UIP pattern ILD and show a progressive fibrosing phenotype, do not fit the definition of IgG4-RRD with a benign prognosis. The lung lesion in our patient, who suffered from IgG4-RD of the submandibular glands, progressively deteriorated-a clinical course that was compatible with UIP pattern ILD, such as IPF, and which also indicated that it was less likely to be IgG4-RRD. The patient has not been receiving corticosteroid treatment according to IPF. Even if steroid treatment is performed in the future, it is highly possible that the reticular shadow will remain or deteriorate after the initiation of such treatment, as is the case with IgG4-positive IP. If the lung lesion of the patient was indeed lung involvement of IgG4-RD, the lesion might have been excluded from lung involvement of IgG4-RD based on the recent criteria. Thus, the lung lesion of the patient should be treated as a different category from conventional IgG4-RRD. We speculate that the patient's lung lesion would have eventually more closely resembled UIP pattern ILD that developed independently of IgG4-RD than lung involvement of IgG4-RD. In the not-so-distant future, the use of anti-fibrotic drugs, such as nintedanib, may be considered for such cases.

In summary, the lung lesion of the patient with IgG4related chronic sclerosing sialadenitis corresponded to definite according to the proposed diagnostic criteria for IgG4RRD; however, the clinical course of this lung lesion was consistent with UIP pattern ILD, such as IPF, and differed from conventional IgG4-RRD. Thus, the lung lesion of our patient should be treated as a different category from IgG4RRD, such as IgG4-positive IP. Our findings suggest that UIP pattern ILD may not be suitable for lung lesions of IgG 4-RD. We propose excluding lung lesions with UIP pattern ILD, especially in cases presenting with the progressive fibrosing phenotype, from IgG4-RRD (10).

\section{The authors state that they have no Conflict of Interest (COI).}

This report was supported by the Ministry of Health, Labor and Welfare Research Program on Rare and Intractable Diseases (No. JPMH20FC1040), the Japan Society for the Promotion of
Science (No.21K08175).

\section{References}

1. Umehara H, Okazaki K, Masaki Y, et al. Comprehensive diagnostic criteria for IgG4-related disease (IgG4-RD), 2011. Mod Rheumatol 22: 21-30, 2012.

2. Umehara H, Okazaki K, Kawa S, et al. The 2020 revised comprehensive diagnostic (RCD) criteria for IgG4-RD. Mod Rheumatol 31: 529-533, 2021.

3. Wallace ZS, Naden RP, Chari S, et al. The 2019 American College of Rheumatology/European League Against Rheumatism classification criteria for IgG4-related disease. Ann Rheum Dis 79: 77-87, 2020 .

4. Stone JH, Zen Y, Deshpande V. IgG4-related disease. N Engl J Med 366: 539-551, 2012.

5. Inoue $D$, Zen $Y$, Abo H, Gabata $T$, et al. Immunoglobulin G4related lung disease: CT findings with pathologic correlations. Radiology 251: 260-270, 2009.

6. Matsui S, Hebisawa A, Sakai F, et al. Immunoglobulin G4-related lung disease: clinicoradiological and pathological features. Respirology 18: 480-487, 2013.

7. Raghu G, Remy-Jardin M, Myers JL, et al. Diagnosis of idiopathic pulmonary fibrosis. An official ATS/ERS/JRS/ALAT clinical practice guideline. Am J Respir Crit Care Med 198: e44-e68, 2018.

8. Travis WD, Costabel U, Hansell DM, et al. An official American Thoracic Society/European Respiratory Society statement: update of the international multidisciplinary classification of the idiopathic interstitial pneumonias. Am J Respir Crit Care Med 188: 733-748, 2013.

9. Flaherty KR, Wells AU, Cottin V, et al. Nintedanib in progressive fibrosing interstitial lung diseases. N Engl J Med 381: 1718-1727, 2019.

10. Matsui S, Yamamoto H, Minamoto S, et al. Proposed diagnostic criteria for IgG4-related respiratory disease. Respir Investig 54: 130-132, 2016.

11. Sun $X$, Liu H, Feng R, et al. Biopsy-proven IgG4-related lung disease. BMC Pulm Med 16: 20, 2016.

12. Kang J, Park S, Chae EJ, et al. Long-term clinical course and outcomes of immunoglobulin G4-related lung disease. Respir Res 21: 273, 2020.

13. Komatsu M, Yamamoto H, Matsui S, et al. Clinical characteristics of immunoglobulin G4-positive interstitial pneumonia. ERJ Open Res 7: 00317-2021, 2021.

14. Wallace ZS, Naden RP, Chari S, et al. Members of the ACR/EULAR IgG4-RD Classification Criteria Working Group. The 2019 American College of Rheumatology/European League Against Rheumatism classification criteria for IgG4-related disease. Ann Rheum Dis 79: 77-87, 2020.

15. Umehara H, Okazaki K, Kawa S, et al. Research Program for Intractable Disease by the Ministry of Health, Labor and Welfare (MHLW) Japan. The 2020 revised comprehensive diagnostic (RCD) criteria for IgG4-RD. Mod Rheumatol 31: 529-533, 2021.

The Internal Medicine is an Open Access journal distributed under the Creative Commons Attribution-NonCommercial-NoDerivatives 4.0 International License. To view the details of this license, please visit (https://creativecommons.org/licenses/ by-nc-nd/4.0/).

(C) The Japanese Society of Internal Medicine Intern Med Advance Publication 